\title{
Effects of Periconceptual Omega-3-Fatty Acid Supplementation on in Vitro Fertilization Success and Miscarriage Rates in Patients of a German Fertility Centre
}

\author{
Arasch Bareksei ${ }^{1,}$, Gerd Hafner ${ }^{2}$, Sebastian Pfeiffer ${ }^{3}$, Kathrin Schlatterer ${ }^{4, ~ *}$ \\ ${ }^{1}$ Dental Concept, Praxis für Zahn, Mund-Und Kieferheilkunde, Voerde, Germany \\ ${ }^{2}$ Institut für Laboratoriumsmedizin, Mikrobiologie Und Hygiene, Evangelisches Klinikum Bethel, Bielefeld, Germany \\ ${ }^{3}$ Labor Todorov, Düsseldorf, Germany \\ ${ }^{4}$ Departments of Dermatology, Venereology, Allergology and Immunology, Dessau Medical Center, Brandenburg Medical School Theodor \\ Fontane, Dessau, Germany
}

\section{Email address: \\ barekseia@aol.com (A. Bareksei), gerd.hafner@evkb.de (G. Hafner), immudoc@hotmail.de (S. Pfeiffer), \\ k.schlatterer@gmx.de (K. Schlatterer) \\ ${ }^{*}$ Corresponding author}

\section{To cite this article:}

Arasch Bareksei, Gerd Hafner, Sebastian Pfeiffer, Kathrin Schlatterer. Effects of Periconceptual Omega-3-Fatty Acid Supplementation on in Vitro Fertilization Success and Miscarriage Rates in Patients of a German Fertility Centre. International Journal of Clinical and Experimental Medical Sciences. Vol. 5, No. 5, 2019, pp. 62-66. doi: 10.11648/j.ijcems.20190505.11

Received: September 6, 2019; Accepted: September 27, 2019; Published: October 10, 2019

\begin{abstract}
Long-chain omega-3 fatty acids have been positively related with improvement of fertility in both women and men. Primary natural source of omega-3- fatty acids is seafood. As this also can bei the source of toxicants like f.e. lead, mercury and cadmium, reproductive benefits may be counteracted. Therefore, our approach was to supplement pharmaceutical grade omega-3-fatty acids in order to assess potential benefits of omega-3-fatty acids on modulation of IVFsuccess by dietary measures in a well-defined group of patients of a German Fertility Centre. A group of 52 IVF-patients with a history of at least two miscarriages or unsuccessful in vitro fertilization approaches received periconceptual omega3-fatty acid supplementation for a time period of at least 3 weeks. The "historical" control group, consisting of 1109 patients without omega-3-fatty acid supplementation, was generated out of the Fertility Centre's patient database. The same inclusion criteria (history of at least two miscarriages or unsuccessful in vitro fertilization approaches) were applied for both groups. Omega-3-fatty acid supplementation lead to significantly enhanced in vitro fertilization success rates as well as to reduction of miscarriage rates compared to patients of the control group. The results encourage to design further agematched, double-blinded prospective studies in order to verify a positive influence of dietary intervention and lifestyle modification on fertility rates.
\end{abstract}

Keywords: Omega-3-Fatty Acid Supplementation, In Vitro Fertilization, Miscarriage Rates

\section{Introduction}

Omega-3-fatty acids belong to the polyunsaturated fatty acids, which naturally occur in different plants, seagrass and fish. As they are essential for humans, they must be supplied by food. They not only represent important structural components of the cell wall but are also essential for synthesis of intra- as well as extracellular signalling transmitters like f.e. eicosanoids. Eicosanoids play an important role in physiological as well as pathological processes like inflammation, tumorigenesis and others.

Postulated effects of omega-3-fatty acid supplementation are quite diverse, such like f.e. prevention of cardiac ischemia, stroke, depression and dementia. This just represents a small selection of positive effects attributed to this class of substances and explains why readiness for supplementation within the population is high. Adaptions in the healthcare market are a direct result.

The question, whether these postulated benefits can be 
scientifically substantiated, triggers a broad variety of studies.

Indeed, some of the effects described are proofed by several studies. Omega-3-fatty acids are capable to reduce cardiovascular risks [1-2], have positive effects on mental health [3] and seem to be associated with anti-inflammatory effects [4]. In some studies, they even are correlated with preventive effects for specific tumour illnesses like prostate cancer [5].

Beside that, there are hints for a positive association of omega-3-fatty acids with male and female fertility [6-10]. Omega-3-fatty acid supplementation increases uterine blood flow, and this seems to facilitate a successful pregnancy. On the other hand, risk of miscarriage rates seems to be reduced, even birth weight of babies [6-8] as well as intelligence quotients [11] seem to be influenced in a positive manner. With respect to pregnant women data suggest that omega-3fatty acid supplementation during pregnancy decreases risk of postpartum depression [12].

Recently published studies discuss a positive correlation of omega-3- and omega-6-fatty acids with increasing implantation probabilities of fertilized eggs after in vitro fertilization [13-14]. Kermack and co-workers postulate that 1 out of 6 couples suffers from subfertility. Despite of improvements in reproductive medicine pregnancy success rates in these specific patient group remain low at $25 \%$ [15].

With a growing knowledge of the potential importance of the periconceptual nutrition status for fertility as well as for a favourable health impact on their progenies a lucrative lifestyle industry has developed in order to cover these demands.

Standardized alimentary supplementation of omega-3-fatty acids before and during an in vitro fertilization therapy so far has not been subject of a well-designed randomized prospective study. Just few small, prospective cohort studies have examined the relationship between serum omega-3-fatty acid levels and IVF-success with conflicting results [16-20]. One of the first approaches to study the correlation of omega3-fatty acid supplementation and the probability of a pregnancy is the PREPARE-trial by Kermack and co-workers [15], which has not been finished yet.

These approaches encouraged us to use the availability of an excellently characterized German Fertility Centre patient group in order to examine the effects of a periconceptual omega-3-fatty acid supplementation on fertility, especially on the probability of pregnancy inception, on the course of pregnancy and on miscarriage rates, in a first preliminary trial.

For this purpose, patients received periconceptual treatment with omega-3-fatty acids over a time-period of at least 3 weeks. Data of the control group were recruited retrospectively by means of the Fertility Centre's database. Inclusion criteria for both groups were either at least 2 miscarriages or non-successful in vitro fertilizations in the past. Therefore, both groups they can be regarded as negatively preselected entities with respect to successful pregnancies after IVF-therapy.

This study approach has been chosen consciously although criteria of a classical prospective study are not fulfilled. It can serve as a first estimation of such an effect in a preselected patient group with respect to negative preconditions.

\section{Material and Methods}

\subsection{Patients}

Patients of a German Fertility Centre (Interdisziplinäres IVF-Zentrum Düsseldorf, Germany) with at least 2 miscarriages or non-successful IVF-therapies were included in the study. This was a patient group preselected with respect to negative preconditions for successful in vitro fertilization therapy. Patients with prednisolonetherapy and patients rejecting disclosure of their data were excluded.

For the retrospective "historical" control group identic criteria were applied. A total of 52 patients with omega-3fatty acid supplementation could be included, 1109 patients without supplementation displayed the control group.

\subsection{Omega-3-Fatty Acid Supplementation}

For the first 3 weeks patients received $300 \mathrm{mg}$ EPA and $200 \mathrm{mg}$ DHA per day. Around the time of conception additionally a weekly intravenous administration of Omegaven ${ }^{\circledR}$ Fresenius emulsion $(1 \mathrm{ml}$ per $\mathrm{kg}$ weight per day) occurred. The manufacturer's declaration characterizes the following concentrations: per $100 \mathrm{ml}$ emulsion $10,0 \mathrm{~g}$ deep sea fish oil, consisting of eicosapentaenoic acid 1.25-2.82 g, docosahexaenoic acid 1.44-3.09 g, $\alpha$-tocopherol 0.015-0.0296 g, besides glycerol (2.5 g) and (3-sn-phosphatidyl-) choline $1.2 \mathrm{~g}$. On average first control of IVF-success was performed 4-6 weeks after supplementation, the shortest control interval was 3 weeks after supplementation.

\subsection{Statistics}

Statistical analysis was performed by means of IBM SPSS Statistics (version 22). Stochastic independency of IVFsuccess and omega-3-fatty acid supplementation was determined by the Chi-Square Independence Test.

\section{Results}

\subsection{Impact of Omega-3 Fatty Acid Supplementation on IVF-Success Rates}

Probabilities of pregnancy occurrence after in vitro fertilization therapy were compared between 52 patients, receiving periconceptual omega-3-fatty acid supplementation, and 1109 patients without supplementation (Table 1). 
Table 1. Comparison of IVF-success rates between omega-3-fatty acid supplemented (n=52) and non-supplemented patients $(n=1109)$ of a German Fertility Centre.

\begin{tabular}{lllllll}
\hline & \multicolumn{2}{l}{ supplemented group } & "historical“ control group & total & \\
\cline { 2 - 7 } & absolute number & $\mathbf{\%}$ & absolute number & $\mathbf{\%}$ & absolute number & \% \\
\hline IVF-success & 36 & $69.2^{* * *}$ & 369 & 33.3 & 405 & 34.9 \\
no IVF-success & 16 & 30.8 & 740 & 66.7 & 756 & 66.1 \\
total & 52 & 100 & 1109 & 100 & 1161 & 100 \\
\hline
\end{tabular}

$* * * \mathrm{p}<0.001$

36 patients of the 52 supplemented became pregnant after the average observation time of 4-6 weeks $(69.2 \%)$. One of these 36 patients suffered from a miscarriage within the further course of pregnancy.

The pregnancy rate within the 1109 patient-control group without supplementation was 369 (33.3\%). The difference between both groups was statistically significant $(\mathrm{p}<0.001)$.

\subsection{Impact of Omega-3 Fatty Acid Supplementation on Miscarriage Rates}

After successful in vitro fertilization therapy 1 out of 32 omega-3-fatty acid supplemented patients $(2.8 \%)$ and 74 out of 369 patients of the control group (20.1\%) suffered from a miscarriage before week 12 (Table 2). The difference between both groups was statistically significant $(\mathrm{p}=0.001)$.

Table 2. Comparison of miscarriage rates between omega-3-fatty acid supplemented ( $n=52$ ) and non-supplemented patients ( $n=1109)$ of a German Fertility Centre.

\begin{tabular}{|c|c|c|c|c|c|c|}
\hline & \multicolumn{2}{|c|}{ supplemented group } & \multicolumn{2}{|c|}{ "historical" control group } & \multicolumn{2}{|l|}{ total } \\
\hline & absolute number & $\%$ & absolute number & $\%$ & absolute numberl & $\%$ \\
\hline miscarriage rate before 12 th week & 1 & $2.8 * * *$ & 74 & 20.1 & 75 & 18.5 \\
\hline persisting pregnancy after 12 th week & 35 & 97.2 & 295 & 79.9 & 330 & 81.5 \\
\hline total & 36 & 100 & 369 & 100 & 405 & 100 \\
\hline
\end{tabular}

$* * * \mathrm{p}=0.001$

\section{Conclusion}

Goal of this preliminary study was to examine if there are first hints for favourable effects of omega-3- fatty acid supplementation in a, with respect to pregnancy probability negatively selected, patient group of a German fertility centre. Endpoints were in vitro fertilization success and miscarriage rates after IVF. Clear selection criteria led to inclusion of 52 patients in the study, while 1109 patients could be included in the "historical" control group. Due to the specific concept of the study and the fact that the control group was nearly 20 times larger than the supplemented group data interpretation is limited, but important hints as basis for further investigations can be deduced.

In the interventional branch of the study successful IVF rates nearly doubled compared to the control group. This promising result is in accordance with shortly published data. Jungheim and co-workers described a positive effect of modulation of alimentary omega-3 vs. omega- 6 fatty acid ratios on IVF-success [14]. Interestingly, animal studies with show that a reduction of omega-3 polyunsaturated fatty acids in aquatic feed reduces sperm competitiveness of freshwater guppies [21].

Further findings in the field of veterinary medicine reinforce the hypothesis of positive effects of omega-3- fatty acids on fertility. Beside guppies, specific carp species benefit from omega-3- and omega-6-fatty acid supplementation with respect to maturation of gonads, spawning and breeding [22]. Pre-pubertal female sheep show an increased release of reproductive hormones and higher fertility in adulthood [23], the success rate of artificial insemination and folliculogenesis in dairy cows is also found to be increased [24]. Omega-3 and omega- 6 fatty acids are described to exert positive effects on fertility in poultry and pigs [25-26].

Conclusively, data available so far on human and animal investigations suggest that supplementation of omega-3-fatty acids in male [27-29] as well female individuals [16, 19-20] improves reproductive success. Therefore, an omega-3-fatty acid supplementation as part of alimentary modification should be considered to improve female as well as male fertility in a multimodal approach.

With respect to miscarriage rates our study reveals first promising results. Within the group of 36 patients in the omega-3-fatty acid supplementation group, who became pregnant, only one suffered from a miscarriage before pregnancy week $12(2.8 \%)$, miscarriage rates in the control group were $20.1 \%$. Despite of the significant difference in both groups these data should be interpreted with caution and confirmed by further investigations due to the different sizes of both study groups and the relatively small number of patients in the interventional study branch.

On the other hand it can be taken into consideration that omega-3-fatty acids so far have been attributed preventive effects with respect to miscarriages before. Lazzarin published data for women suffering from repetitive miscarriages due to limited uterine perfusion. Alimentary supplementation of omega-3-fatty acids revealed a significant improvement of arterial uterine circulation [30]. Women with anti-phospholipid syndrome-induced miscarriages also benefitted from omega-3-fatty acid fish oil supplementation resulting in a reduction of miscarriage incidences [20]. 
Supplementation of specific vitamins, omega-3-fatty acids and minerals reduced the risk of preeclampsia, premature birth, gestational diabetes and miscarriages [31]. In parallel, veterinary medicine data suggest that miscarriage rates in dairy cows, receiving food blended with omega-3-fatty rich linseed, can be halved [32].

Results of our study in combination with the data available so far encourage to attribute a potential beneficial effect of omega-3-fatty acid supplementation on IVF-success and on miscarriage rates after IVF therapy.

Certainly to be emphasized in our study is the fact that all 52 patients in the treatment branch as well as the 1109 patients in the control branch were excellently characterised and monitored over a long period. By a negative preselection with respect to at least 2 miscarriages or 2 failed IVF treatments this group compositions clearly differ from the average woman of childbearing age. It has to be discussed if, due to the negative preselection of the study group, beneficial effects of dietary measures in the average female population possibly could be even more significant. This is to be evaluated in further well-designed, age-matched prospective studies.

Interpretation of our results is limited since the study could not be performed as a classic interventional trial. The prospective interventional branch had to be compared with a retrospective control branch, both also differing significantly in size. The relatively small sample size of omega-3-fatty acid supplemented fertility centre patients could have favoured the statistically significant $p$-values by stochastically fluctuations. Beside this, age-matching was not possible in the study design chosen. Promising preliminary results of this study together with data available so far encourage to design further age-matched, placebo-controlled double-blinded trials. The importance of such an approach is pointed out by the ongoing PREPARE-trial [15]. This randomized placebo-controlled concept is based on a 6-week olive-oil and omega-3-fatty acid nutritional supplementation combined with vitamin D administration in the interventional branch in order to examine if dietetic intervention is capable to positively influence early stages of embryonic development.

In our study first beneficial effects of a periconceptual omega-3-fatty acid supplementation on patients of a German Fertility Centre with respect to IVF-success and reduction of miscarriage rates could be detected. If these findings can be substantiated in further studies, this life-style modification could become an important and cost-effective component in improvement of fertility therapies. Furthermore, discussion can be encouraged if appropriate recommendations for all women at childbearing age are justified at this point.

\section{References}

[1] Burr, M. L., Fehily, A. M., Gilbert, J, F., Rogers S., Holliday R, M., Sweetnam P, M. et al. (1989) Effects of changes in fat, fish, and fibre intakes on death and myocardial reinfarction: diet and reinfarction trial (DART). Lancet, 2, 757-761.
[2] Marchioli, R., Barzi, F., Bomba, E., Chieffo, C., Di, G. D., Di, M. R. et al. (2002) Early protection against sudden death by n3 polyunsaturated fatty acids after myocardial infarction: timecourse analysis of the results of the Gruppo Italiano per lo Studio della Sopravvivenza nell'Infarto Miocardico (GISSI) Prevenzione. Circulation, 105, 1897-1903.

[3] Freeman, M. P., Hibbeln, J. R., Wisner, K. L., Davis, J. M., Mischoulon, D., Peet, M. et al. (2006) Omega-3 fatty acids: evidence basis for treatment and future research in psychiatry. Journal of Clinical Psychiatry, 67, 1954-1967.

[4] Yan Y., Jiang W., Spinetti T., Tardivel A., Castillo R., Bourquin C. et al. (2013) Omega-3 fatty acids prevent inflammation and metabolic disorder through inhibition of NLRP3 inflammasome activation. Immunity, 38, 1154-1163.

[5] Chavarro, J. E., Stampfer, M. J., Li, H., Campos, H., Kurth, T., Ma, J. (2007) A prospective study of polyunsaturated fatty acid levels in blood and prostate cancer risk. Cancer Epidemiology, Biomarkers \& Prevention, 16, 1364-1370.

[6] Honest, H., Forbes, C. A., Duree, K. H., Norman, G., Duffy, S B., Tsourapas, A. et al. (2009) Screening to prevent spontaneous preterm birth: systematic reviews of accuracy and effectiveness literature with economic modelling. Health Technology Assessment, 13, 1-627.

[7] Nakao, J., Ohba, T., Takaishi, K., Katabuchi, H. (2015) Omega-3 fatty acids for the treatment of hypertriglyceridemia during the second trimester. Nutrition, 31, 409-412.

[8] Di Cintio, E., Parazzini, F., Chatenoud, L., Sourace, M., Benzi, G., Zanconato, G. et al. (2001) Dietary factors and risk of spontaneous abortion. European Journal of Obstetrics \& Gynecology and Reproductive Biology, 95, 132-136.

[9] Salas-Hueto, A., Rosique-Esteban, N., Becerra-Tomás, N., Vizmanos, B., Bulló, M., Salas-Salvadó, J. (2018) The effecst of nutrients and dietary supplements on sperm quality parameters: a systematic review and meta-analysis of randomized clinical trials. Advances in Nutrition, 9, 833-848.

[10] Falsig, A. L., Gleerup, C. S., Knudsen, U. B. (2019) The influence of omega-3 fatty acids on semen quality markers: a systematic PRISMA review. Andrology, doi: 10.1111/andr.12649. [Epub ahead of print].

[11] Helland, I. B., Smith, L., Saarem, K., Saugstad, O. D., Drevon, C. A. (2003) Maternal supplementation with very-long-chain n-3 fatty acids during pregnancy and lactation augments children's IQ at 4 years of age. Pediatrics, 111, e39-e44.

[12] Jensen, C. L. (2006) Effects of n-3 fatty acids during pregnancy and lactation. The American Journal of Clinical Nutrition, 83, 1452S-1457S.

[13] Mmbaga, N., Luk, J. (2012) The impact of preconceptual diet on the outcome of reproductive treatments. Current Opinion in Obstetrics and Gynecology, 24, 127-131.

[14] Jungheim, E. S., Frolova, AI., Jiang, H., Riley, J. K. (2013) Relationship between serum polyunsaturated fatty acids and pregnancy in women undergoing in vitro fertilization. The Journal of Clinical Endocrinology and Metabolism, 98, E1364-E1368.

[15] Kermack, A. J., Calder, P. C., Houghton, F. D., Godfrey, K. M., Macklon, N. S. (2014) A randomised controlled trial of a periconceptional dietary intervention in women undergoing IVF treatment (PREPARE trial). BMC Women's Health, 14, 130. 
[16] Lass, A:, Belluzzi, A. (2019) Omega-3 polyunsaturated fatty acids and IVF treatment. Reproductive Biomedicine Online, $38,95-99$.

[17] Eskow, A. M., Wormer, K. C., Matthews, M. L., Norton, H. J. Papadakis, M. A., Hurst, B. S. (2017) The association between fatty acid index and in vitro fertilization outcomes. Journal of Assisted Reproduction and Genetics, 34, 1627-1632.

[18] Wise, L. A., Wesselink, A. K., Tucker, K. L., Saklani, S., Mikkelsenm E. M., Cueto, H., Riis, A. H. et al. (2017) Dietary fat intake and fecundability in 2 preconception cohort studies. American Journal of Epidemiology, 187, 60-74.

[19] Mumford, S. L., Browne, R. W., Kim, K., Nichols, C., Wilcox, B., Silver, R. M., Connell, M. T. et al. (2018) Preconception plasma phospholipid fatty acids and fecundability. The Journal of Clinical Endocrinology \& Metabolism, 103, 45014510 .

[20] Chiu, Y. H., Karmon, A. E., Gaskins, A. ., Arvizu, M., Williams, P. L., Souter, I., Rueda, B. R. et al. (2018) Serum omega-3 fatty acids and treatment outcomes among women undergoing assisted reproduction. Human Reproduction, 33, 156-161.

[21] Rahman, M. M., Gasparini, C., Turchini, G. M., Evans, J. P. (2014) Experimental reduction in dietary omega-3 polyunsaturated fatty acids depresses sperm competitiveness. Biology Letters, 10, 20140623.

[22] Nandi, S., Chattopadhyay, D. N., Verma, J. P., Sarkar, S. K., Mukhopadhyay, P. K. (2001) Effect of dietary supplementation of fatty acids and vitamins on the breeding performance of the carp Catla catla. Reproduction Nutrition Development, 41, 365-375.

[23] Ghaffarilaleh, V., Fouladi-Nashta, A., Paramio, M. T. (2014) Effect of alpha-linolenic acid on oocyte maturation and embryo development of prepubertal sheep oocytes. Theriogenology, 14, 82, 686-696.

[24] Moallem, U., Shafran, A., Zachut, M., Dekel, I., Portnick, Y.,
Arieli, A. (2013) Dietary alpha-linolenic acid from flaxseed oil improved folliculogenesis and IVF performance in dairy cows, similar to eicosapentaenoic and docosahexaenoic acids from fish oil. Reproduction, 146, 603-614.

[25] Lee, S. A., Whenham, N., Bedford, M. R. (2019) Review on docosahexaenoic acid in poutry and swine nutrition: consequences of enriched animal products on performance and health characteristics. Animal Nutrition, 5, 11-21.

[26] Salas-Huetos, A., James. E. R., Aston, K. I., Jenkins, T. G., Carrell D. T. (2019) Diet and sperm quality: nutrients, foods and dietary patterns. Reproductive Biology, doi: 10.1016/j.repbio.2019.07.005. [Epub ahead of print].

[27] Yao, D., Mills, J. N. (2016) Male infertility: lifestyle factors and holistic, complementary, and alternative therapies. Asian Journal of Andrology, 18, 410-418.

[28] Minguez-Alarcón, L., Chavarro, J. E., Mendiola, J., Roca, M., Tanrikut, C., Vioque, J., Jorgensend N. et al. (2017) Fatty acid intake in relation to reproductive hormones and testicular volumen among young healthy men. Asian Journal of Andrology, 19, 184-190.

[29] Lazzarin, N., Vaquero, E., Exacoustos, C., Bertonotti, E., Romanini, M. E., Arduini, D. (2009) Low-dose aspirin and omega-3 fatty acids improve uterine artery blood flow velocity in women with recurrent miscarriage due to impaired uterine perfusion. Fertility and Sterility, 92, 296-300.

[30] Rossi, E., Costa, M. (1993) Fish oil derivatives as a prophylaxis of recurrent miscarriage associated with antiphospholipid antibodies (APL): a pilot study. Lupus, 2, 319-323.

[31] Glenville, M. Nutritional supplements in pregnancy: commercial push or evidence based? (2006) Current Opinion in Obstetrics and Gynecology, 18, 642-647.

[32] Ambrose, D. J., Kastelic, J. P., Corbett, R., Pitney, P. A., Petit, H. V., Small, J. A. et al. (2006) Lower pregnancy losses in lactating dairy cows fed a diet enriched in alpha-linolenic acid. Journal of Dairy Science, 89, 3066-3074. 